\title{
Genetic modification of Mammalian Genome at Chromosome level ${ }^{*}$
}

\author{
OLEG L. SEROV ${ }^{1,2}$, NATALIA M. MATVEEVA ${ }^{2}$, \\ IRINA A. SEROVA ${ }^{1,2}$ and PAVEL M. BORODIN ${ }^{1,2}$ \\ ${ }^{1}$ Institute of Biophysics, Federal University of Rio de Janeiro, \\ 21949-900 - Rio de Janeiro, Brazil \\ ${ }^{2}$ Institute of Cytology and Genetics, Novosibirsk 630090, Russia \\ Manuscript received on May 11, 2000; accepted for publication on May 17, 2000; \\ presented by A. C. CAMPOS-DE-CARVALHO**
}

\begin{abstract}
The review is concerned with a progress in genetic modification of a mammalian genome in vitro and in vivo at chromosomal level. Recently three new approaches for the chromosome biotechnology have been developed: Using Cre/loxP-system a researcher is able to produce targeted rearrangements of whole chromosomes or their segments or particular genes within the genome, and therefore to modify the set, position and copy number of the endogenous elements of the genome. Mammalian artificial chromosomes (MACs) provide a possibility to introduce into genome relatively large segments of alien chromosome material, either artificially constructed or derived from the genome of different species. Using ES-somatic cell hybrids allows to transfer whole chromosomes or their fragments between different genomes within and between species. Advantages and limitations of these approaches are discussed.
\end{abstract}

Key words: chromosome biotechnology, Cre/loxP, ES cells, MAC.

\section{INTRODUCTION}

Genetic modification of a mammalian genome is the most fascinating technology developed in the last two decades. In fact, now this technology allows one to manipulate with genetic materials in vivo from individual genes (in case of transgenesis) to whole genome(s) for cloning of animals. For a long time transgenic animal technology was a single approach for genetic modification of a mammalian genome (Chan 1999). Classic variant of transgenesis relies on microinjection of exogenous DNA into the pronuclei of the zygotes. Then the injected DNA can be incorporated into the recipient genome

\footnotetext{
*Invited paper

** Member of the Academia Brasileira de Ciências

Correspondence to: O. L. Serov

E-mail: serov@biof.ufrj.br
}

(Palmiter \& Brinster 1986, Gordon 1997). The incorporated exogenous DNA ("transgene") is able to be inherited and, i.e., to be transmitted in the next generation so that to produce animals with novel gene(s). The approach provided a fast progress in the study of gene function and regulation of development (Palmiter \& Brinster 1986, Chan 1999). Initially, the approach was applied to mice only but in a short time it became used in the experiments with farm animals as well (Hammer et al. 1985, Chan 1999). However, the DNA injected via pronuclear microininjection is usually been integrated into the recipient genome in a random manner. For this reason, expression of transgene varies in a wide range among transgenic animals. Presumably this variation depends upon a site of integration ("position effect") or copy number. 
This problem was solved when of the gene targeting approach has been developed (Thomas \& Capecchi 1987). This approach allows one to introduce an in vitro modified gene via homologous recombination between exogenous DNA and targeting chromosome DNA. This method is in of great use in genome modification of pluripotent embryonic stem (ES) cells. ES cells with modified targeted gene can be combined with early embryos to produce chimeras (see below) which, in turn, are able to generate offspring carrying the modified targeted gene (Robertson 1987). The gene targeting has opened the gate to a new era in transgenic technology named as a site-directed mutagenesis or "knock out" technology (Capecchi 1989). Numerous publications on "knock-out" mice have appeared in the last decade. These animals are in a great use as a tool to studies in gene regulation, as well as in modeling human inherited diseases.

In spit the progress in transgenic technology, development of the technology for making largescale alternations to the mammalian genome including manipulation with whole or fragments of chromosomes in vivo was yet to come. Five years ago such technology has been successfully developed and applied to ES cells in vitro (Smith et al. 1995, van Deursen et al. 1995) and even in vivo (RaminezSolis et al. 1995). The technology may be named as chromosome engineering (Raminez-Solis et al. 1995) or chromosome biotechnology. More recently two new approaches for the chromosome biotechnology have been developed: one is transfer of whole chromosome or its fragments into a mammalian genome by ES-cell vector, and another is the creating mammalian artificial chromosomes.

The review is concerned with a progress in genetic modification of a mammalian genome in vivo at chromosomal level.

\section{Cre/loxP-MEDIATED GENERATION OF SITE-SPECIFIC INTRA- AND INTER- CHROMOSOMAL REARRANGEMENTS IN MAMMALIAN GENOME}

Recent development of a new sophisticated approach based on use of Cre-loxP system has revo- lutionized the transgenic biotechnology of animals (Metzger \& Feil 1999). The system functions as following manner. The recombination is initiated by $\mathrm{Cre}$ proteins (cyclization recombinase) binding to 13-bp inverted repeats in the loxP sites (a size of loxP is $34 \mathrm{bp}$ ) and promoting synapsis (or joining) of a pair of sites. Cre proteins catalyze exchange between the pair of sites within an asymmetric 8-bp central spacer sequence of $\operatorname{lox} P$ site by concerted cleavage and rejoining reactions. The recombination between two loxP sites inserted in opposite orientation leads to inversion. In case of recombination between two $\operatorname{lox} P$ sites inserted in direct orientation results in deletion or translocation. Thus, the Crelox $P$-system provides a site-specific recombination, and it is predictable on the basis of the relative orientations of the loxP sites.

Remarkable merits of the Cre-loxP system was firstly in transgenesis. Site-specific recombination mediated by Cre-loxP approach allows researchers to control not only site-specific integration and copy number of transgene but also to replace or delete precisely any sequence within a targeting gene (Gu et al. 1994, Araki et al. 1995, Lasko et al. 1996, Babinet 1997). Moreover, the site-specific recombination mediated by $\mathrm{Cre}$-loxP has been used to manipulate gene expression in a cell type specific or inducible manner at the stage of development, which need to be investigated (Schwenk et al. 1995, Babinet 1997, Metzger \& Feil 1999).

The Cre/loxP system was somewhat latter applied to induce a variety of chromosome rearrangements in ES cells that can be selected and than used for produce ES-derived mice (Smith et al. 1995, van Deursen et al. 1995, Raminez-Solis et al. 1995). Cre/loxP-mediated intra-and interchromosomal rearrangements can generate large deletions, inversions, duplications, and translocations.

A general strategy to produce Cre/loxPmediated site-directed chromosomal rearrangements can be illustrated by an approach developed by Raminez-Solis et al. (1995). The authors used, as a selectable gene marker, the HPRT minigene (hypoxanthine phosphoribosyltransferase) fused to 
a $P G K$ promoter (phosphoglycerate kinase-1 gene), intron 2 of HPRT gene and poly $\left(A^{+}\right)$signal. The $H P R T$-minigene was modified by the insertion of a loxP site in the HPRT intron. This construct is cleaved into two parts; one contained the $P G K$ promoter, the exons 1 and 2, the $\operatorname{lox} P$ - intron 2, and HPRT exons 3-6 whereas another contained exons 3-9 and poly $\left(A^{+}\right)$signal. Thus, both secondary constructs contained a $2 \mathrm{~kb}$ overlap including the loxP site. Independently these parts do not provide HATresistance after transfection of HPRT-deficient ES cells. Each of the constructs was fused to positive selectable marker either the $n e o^{+}$-resistance or puromycin-resistance gene to prepare of two positive selectable cassettes. Both types of positive selectable cassettes were ligated to the large genomic fragments (more than $5 \mathrm{~kb}$ ) complementary to the genes selected for gene targeting. Raminez-Solis $e t$ al. (1995) chose for gene targeting the gene pairs located in mouse chromosome 11 with different distances relatively to each others: the Hoxb1 and $H o x b 9$ (a distance between the genes is $90 \mathrm{~kb}$ ), the $G s$ and the $E_{2} D H$ (a distance between the genes is $1.0 \mathrm{Mb}$ ), the $E_{2} D H$ and $W n t$ (a distance between the genes is 3-4 cM), and the Hoxb cluster and the $E_{2} D H$ (a distance is 3-4 cM). Both resulting cassettes were used for transfection of the HPRTdeficient ES cells. After two consecutive rounds of transfection of the ES cells by these resulting cassettes, double-targeted clones were isolated using a selective media; these clones were $\mathrm{neo}^{+}$- and puromycin-resistance and HAT-sensitive.

To produce the ES cells carrying predicted chromosomal rearrangements, several doubletargeted clones were transiently transfected with a Cre-expression cassette and placed under HAT selection. Transient expression of $\mathrm{Cre}$ induced recombination between the $\operatorname{lox} P$ sites and, as a result, reconstructed a functional HRPT-minigene. The reconstructed HPRT-minigene allowed the HATresistance clones to proliferate. In these clones a rearrangement of chromosome 11 between the loxP sites might be took place. Raminez-Solis et al. (1995) demonstrated that the lox $P$-mediated recom- bination in the double-targeted ES cell clones resulted in series of deletions ranging from $90 \mathrm{~kb}$ to 3-4 cM, inversions or duplications depending on the orientation of the loxP sites. Moreover, four clones carrying the deletion on one homolog and the duplication on another were used for production of chimeric mice by injection of these ES cells into blastocysts. Offspring of some chimeras were hemizygous for this deletion, heterozygous for the duplication or homozygous for the duplication. Most exciting result was that the mice were all fully viable. Thus, the strategy allows to design a variety of chromosome rearrangements in vitro and in vivo by a Cre-loxP-mediated site-directed recombination.

Using similar approach, Smith et al. (1995) have produced a chromosomal translocation between chromosome 15 (where the targeted $c-m y c$ gene with a loxP site was located) and chromosome 12 (where another targeted immunoglobulin heavy chain gene with a loxP site was located) in the double-targeted ES cells after their transfection by transient Cre-expression. With the use of the $\mathrm{Cre}$ loxP system, van Deursen et al. (1995) induced a site-specific translocation between the targeted Dek gene on chromosome 13 and the targeted Can gene on chromosome 2 in mouse ES cells.

Cre-loxP-mediated chromosome rearrangements have been generated not only in vitro in ES cells, but also in vivo. Special deleter strains of transgenic mice have been breed (Schwenk et al. 1995). The strains was produced by injection of a DNA construct into the fertilized eggs. The construct contained the $\mathrm{Cre}$ gene ligated to a cytomegalovirus minimal promoter. Schwenck et al. (1995) tested the transgenic mice in crossing to another transgenic mice carrying an allele of the DNA polymerase $\beta$ gene flanked by loxP sites. All offspring had the deletion of the allele in all tissues. Moreover, back-crossing the $F_{1}$ generation to wildtype mice revealed that the $\mathrm{Cre}$-mediated deletion was present in germ cells since the deletion was transmitted to the offspring. These strains made possible a targeted production of chosen deletions of whole chromosomes, their segments or ever par- 
ticular genes. In fact, Lewandoski \& Martin (1997) have produced mice with genotype $\mathrm{XO}$ by a Crelox $P$-mediated site-specific recombination. In the case, mice carrying two loxP sites in inverted orientation on Y-chromosomes were crossed with the transgenic females carrying a Cre gene that was expressed in the early embryo. Almost all male embryos loss the $\mathrm{Y}$ chromosome in early development. The authors suggested to use the approach as a common method to produce mice with deletion in any chromosomes.

More recently, Herault et al. (1998) suggested a new modification of the Cre-loxP-directed sitespecific system. The authors have produced a transgenic mice carrying $\mathrm{Cre}$-expessing transgene under the transcriptional control of $S y c p 1$ promoter. The gene controls the synaptonemal complex protein-1, which is expressed at an early stage of male meiosis when homologous chromosome pairing occurs. They also generated of two lines of transgenic mice with loxP sites at different positions within the Hoxd complex and crossed them in order to obtain mice having both loci. Both loxP sites were in an orientation opposite to that of the endogenous Hox genes. This genetic construct induced meiotic trans-allelic recombination and two new haplotypes appeared: one with a duplication of Hoxd12 and the other with a deletion of this targeted gene. Essentially the same approach was used by Bunting et al. (1999) for targeted self-excision of Hoxa3 gene in the germ line.

Thus, Cre/loxP-system provides a powerful tool to produce targeted rearrangements of whole chromosomes or their segments or particular genes and by this way to modify structure and function of mammalian genome at large-scale.

\section{MAMMALIAN ARTIFICIAL CHROMOSOMES (MAC) AND CHROMOSOMAL TRANSGENESIS}

Initial motivation for creating artificial chromosomes in Saccharomyces cerevisiae was the need to elucidate the DNA components necessary for chromosome structure and function. Studies in yeast revealed that at least three components were needed to produce a linear, mitotically stable chromosomes: a centromere, two telomeres and origins of replication. This finding open a way to generation of mammalian artificial chromosomes (MACs).

The importance of MACs is obvious. They provide a unique tool to study the relationships between the chromosome structure and its function in mammalian cells, the organization and function of large genes and gene clusters. They also might serve as a tool of chromosomal transgenesis as they could carry relatively large genes or several genes of interest (Brown et al. 2000).

There are two approaches to building MACs: i) to assemble them in vitro from cis-acting functional sequences; ii) to produce microchromosomes and modify them in vivo.

The first approach has been developed by Harrington et al. (1997). They demonstrated that simultaneous transfection of human somatic cells with alpha satellite, telomeric and genomic carrier DNA could lead to de novo formation of wholly functional MACs. The resulting MACs were relatively large: 6-10 Mb, which corresponded to $10-20 \%$ of the size of human chromosomes. These MACs shown to be cytogenetically stable and maintained for dozens of generations in culture.

Series of microchromosomes derived from human $\mathrm{X}$ and $\mathrm{Y}$ chromosomes have been generated using telomere-associated chromosome fragmentation. The fragmentation was achieved due to targeting telomeric DNA to the centromeric array of alphoid DNA in each of the two possible orientations (Farr et al. 1995, Heller et al. 1996). Heller et al. (1996) introduced several human Y-chromosome derived microchromosomes into mouse ES cells. The success was rather limited, however. Only one of the ES cell lines was able to maintain a $4 \mathrm{Mb}$ microchromosome, which had acquired a functional mouse centromere.

The main problem which restricts the use of MACs is that they can only be manipulated by homologous recombination, which is inefficient in human and mouse cell lines. However, it has been shown that lymphoma-derived chicken cell lines 
could be used to overcome this obstacle. Dieken et al. (1996) and Brown et al. (1996) suggested to transfer human microchromosomes or artificially assembled MACs into lymphoma-derived chicken cells by microcell fusion and then modify them by homologous recombination. After that these chromosomes can be transferred to ES cells by microcell fusion. The resulted ES cells containing these modified MACs then can be used to produce chimeric animals.

\section{TRANSFER OF INDIVIDUAL CHROMOSOMES OR THEIR FRAGMENTS INTO THE GENOME VIA ES-SOMATIC CELL HYBRIDS}

High developmental potential is a remarkable and unique feature of ES cells. There are ample data indicating that the ES cells derived from preimplantation embryos (Robertson 1987) or primordial germ cells (Donovan 1994) are able to retain their pluripotency in in vitro culture conditions. The pluripotent capacity of ES cells can easily be revealed by their injection into the blastocoele. In this case, ES cells can contribute to the full range of adult tissues, including the germ line (Papaioannou \& Johnston 1993, Hogan et al. 1994).

This prompted us to take advantage of the developmental potential of ES cells to reprogram single chromosomes derived from differentiated cells (Matveeva et al. 1996, 1998). We chose cell hybridization as a mild method for introduction of intact chromosomes of differentiated cells into the ES cell genome. When we started our fusion experiments of ES cells with splenocytes (Matveeva et al. 1996, 1998), it was unknown whether the ESsomatic cell hybrids would retain the pluripotency of the ES partner after close contact with the somatic genome. However, we were encouraged by previous reports that hybrid cells obtained by fusion of embryonic carcinoma (EC) cells with differentiated cells retain certain pluripotent properties (Miller \& Ruddle 1977, Andrews \& Goodfellow 1980, Gmur et al. 1980, Takagi et al. 1983, Takagi 1988, Serov et al. 1990).

In our experiment of 1996-1997, we isolated 4 clones of hybrid cells which have been obtained by a fusion of the ES cells with the splenocytes of an adult female of DD/c mice. Three clones contained 41-43 chromosomes (at 13-15th passage) whereas one was nearly tetraploid, and all expressed the selectable HPRT marker of the somatic partner. This was unexpected because most hybrid cells obtained so far by fusion of the EC or ES cells with somatic cells showed slow chromosome segregation and were either tetraploid or nearly tetraploid (Takagi et al. 1983, Takagi 1988, Tada et al. 1997).

The 3 hybrid clones with the near diploid karyotype shared features similar to the ES cells (Matveeva et al. 1996). They retained pluripotency including their ability to contribute to the full range of adult tissues (except the germ line, see below) of the chimeras. Moreover, analysis of the chimeras demonstrated that the $\mathrm{X}$ chromosome derived from the somatic partner did not segregate during their development, judging by the retained expression of its HPRT marker gene in many chimeric tissues (Matveeva et al. 1998). It should be emphasized that hybrid cells encounter non-selective conditions when injected into the blastocoele. The weak segregation of the "somatic" X chromosome suggests that the hybrid cell genome may not discriminate it from the "pluripotent" $\mathrm{X}$ chromosome. Thus, the "somatic" $\mathrm{X}$ chromosome in the pluripotent hybrid cells had the opportunity to reprogram and revert to the condition characteristic of the $\mathrm{X}$ of the pluripotent genome. High pluripotent features and evidence of reprogramming at the imprinting loci were observed in hybrid cells obtained by fusion of embryonic germ cells with lymphoid cells (Tada et al. 1997). Summarizing, our data demonstrated that the whole X-chromosome can be transferred from an adult animal into genome another using ES-somatic cell hybrids as a vector.

However, it should be noted that, in our case, there were no mice with the 129/Ola genotype among over 50 chimera offspring (Matveeva et al. 1998). The disbalanced of the sex chromosomes and trisomy of some autosomes are presumably responsible for it. Thus, cells with the XXY consti- 
tution cannot pass through meiosis and give rise to gametes (Endo et al. 1991, Hernandez \& Fisher 1999). Similarly, the trisomy might have decreased the proliferative activity of hybrid cells, which eventually conferred a selective advantage to the cells of the recipient embryo.

The origin of trisomy in ES-somatic hybrid cells is unclear. Analysis of chromosome segregation in intraspecific cell hybrids is difficult because the parental chromosomes are indistinguishable by the usual G-banding protocols. However, a set of microsatellite markers enabling the identification of any pair of homologous chromosomes derived from different stocks of mice is now available (Dietrich et al. 1992, a database from Jackson Laboratory: www.informatics.jax.org). With this in mind, more recently we have performed analysis of microsatellites for some chromosomes of hybrid clones by PCR-assisted methods (Dietrich et al. 1992). The PCR analyses of the hybrid clones demonstrated that they contained the markers for chromosomes 1,11 and 15 of both parental partners, however HM-1-derived markers were predominantly represented whereas those representing DD/c chromosomes were present in amounts comparable to the number of cells with trisomy for these chromosomes (Serov et al. 1999). The data suggest that chromosome segregation of the somatic partner is not complete in the population of the hybrid cells and probably it is a cause of trisomy. However, it should be emphasized that $20-30 \%$ of cells had a diploid karyotype, 38 autosomes and XY at the 20-22 passage. Complete chromosome segregation cannot be excluded in these hybrid cells.

Surprisingly, all hybrid clones after the 20th passage showed the predominant presence of $\mathrm{X}$ chromosome markers derived from $\mathrm{DD} / \mathrm{c}$ and not 129/Ola mice. In addition, a specific probe for the murine $\mathrm{X}$ chromosome painted only a single chromosome in most hybrid cells. Substitution of the X chromosome derived from the ES cells by "the somatic" X might have taken place in the hybrid cells during their long-term culturing in selective medium (Serov et al. 1999).
As mentioned above, it remained unclear whether germ line transmission of the "somatic" $\mathrm{X}$ in chimeras is possible or not: The unbalanced karyotype of the hybrid cells probably does not allow them to pass through meiosis. For this reason, our goal is to produce hybrid cell clones with a diploid karyotype, carrying the $\mathrm{X}$ chromosome derived from the somatic partner. We hope that such hybrid cells obtained by fusion of the ES cells with somatic differentiated cells would serve as vehicles of individual chromosomes from the genome of one individual to another obviating the sexual process.

Recently Tomizuka et al. (1997, 2000) developed another approach to transfer human chromosomes or their fragments into the murine genome by microcell-mediated chromosome transfer (MMCT). A scheme of the approach was following:

i) initially, the researchers have prepared somatic cell hybrids by fusion between human fibroblasts transformed by $n e o^{R}$-selectable gene with murine established A9 cells. The primary hybrid cells contained a human chromosome carrying a selectable marker and served as donor for preparation of microcells;

ii) then, the investigators transferred human chromosomes from the primary hybrid cells into murine ES by microcell-fusion technique (Tomizuka et al. 1997). As a result, they have isolated a set of secondary ES-cell clones containing either human whole chromosome 22 (Hsa22) or fragments of human chromosomes 2 (Hsa2) or 14 (Hsa14);

iii) the resulting ES-cell clones were used to generate chimeras by microinjection the secondary ES-cell into the blastocoele. All ES-cell clones were able to produce chimeras. Moreover, many chimeras showed in most tissues a presence of human chromosomal material. The chimeras were named as trans-chromosomic (Tc) mice;

iv) the Tc chimeras were crossed with nonchimeric mice. Four Tc male chimeras, derived 
from $\mathrm{hCF}(2-\mathrm{W} 23)$ clone (genotype 39,XY) carrying a fragment of Hsa2, produced 316 pups, of which 2 showed in their karyotype a presence of a fragment of Hsa2 (Tomizuka $e t$ al. 1997). Interestingly, among 67 offspring from four $\mathrm{Tc}$ female chimeras derived from the same ES-cell clone, 22 carried a fragment of Hsa2.

The transmission of human chromosomal material was possible through the male and female germ line, although its inheritance appeared to be more efficient through the females. It allowed the researches to establish a Tc W23 line of mice carrying a fragment of $\mathrm{Hsa} 2$ (a size is $\approx 2 \mathrm{Mb}$ ) and to observe its transmission for four generations. Also, it should be noted that chimeras carrying whole Hsa22 and a fragment (a size is $\approx 50 \mathrm{Mb}$ ) of Hsa14 did not transmit the human chromosomal material for the next generations.

More recently Tomizuka et al. (2000) have extended the experiments and produced a new Tc line of mice carrying a small fragment (a size is $\approx$ $1.5 \mathrm{Mb}$ ) of Hsa14. The authors isolated a subclone (A9/SC20) derived from a A9/14-C11hybrid clone obtained in the first fusion between human fibroblasts with mouse A9 cells (see above). A9/14-11 hybrid cells contained approximately one-fifth of an intact Hsa14 and were used as a donor for MMCT into a female murine ES cell line (TT2F cells, genotype $39, \mathrm{XO}$ ). Stability tests under the non-selective condition allowed the researchers to reveal one of the microcell ES hybrid clone (\# 21) retained with high stability a fragment of Hsa14. The fragment of Hsa14 showed the presence of 14cen and 14qter sequences without the presence of murine satellites. Twenty chimeras were obtained by microinjection of \# 21 ES cells into the blastocoele. The mating of two female chimeras with non-chimeric males resulted 10 of 30 offspring carrying a fragment of Hsa14. According to data of FISH and PCR analyses, the fragment of Hsa14 was quite stable transmittable in the next generations (Tomizuka et al. 2000).

The transferred fragments of chromosomes 2 and 14 carried the $I g$ (immunoglobulin) kappa light chain locus (Igk) and the heavy chain locus $(\operatorname{IgH})$, respectively. The loci were expressed in proper tissue-specific manner in adult chimeric tissues (Tomizuka et al. 1997, 2000). Moreover, in the elegant experiments using double-Tc/double-knock out mice, the researchers demonstrated that the somatic cells of the mice retained both fragments of $\mathrm{Hsa} 2$ and Hsa14. A rescue of the defect of $I g$ production was achieved by high level of expression of human $\operatorname{IgH}$ and $I g k$ in the absence of mouse heavy and kappa chains (Tomizuka et al. 2000). In addition, the double-Tc/double-knock out mice were immunized by human serum albumin (HAS). The mice produced antibodies against HAS with high titers. One of the HAS-immunized doubleTc/double-knock out mouse served as a donor for production of splenic hybridomas. Analysis of the resulting hybridoma supernatants demonstrated that the hybridomas were able to produce HAS-specific fully human $\mathrm{IgG} / \mathrm{k}$ antibodies. These data indicated that the double-Tc/double-knock out mice can be used to obtain antigen-specific human monoclonal antibodies with various isotypes. High affinities of the resultant human monoclonal antibodies suggested that the authentic repertoire of fully human Igs was reconstituted in the double-Tc/doubleknock out mice (Tomizuka et al. 2000). Thus, the study demonstrated that MMCT protocol is an efficient tool for introduction of very large amounts of foreign genetic material into mouse genome.

Recently Hernandez et al. (1999) have obtained a set of Tc chimeric mice carrying fragments (sizes from 0.2 to $50 \mathrm{Mb}$ ) of Has21. To produce the Tc animals, Hernandez et al. (1999) introduced a selectable marker into Hsa21 of human somatic cells. Then, the chromosome was transferred into murine ES cells by irradiated microcell-mediated chromosome transfer. The original MMCT (Tomizuka et al. 1997) was modified by irradiation of microcells before fusion with ES cells, in order to fragmentize the targeted Hsa21. Chimeric mice, obtained by injection of the ES-cell hybrids into the blastocoele, maintained the fragments of Hsa21 and expressed 
the genes of the human chromosome in many tissues.

\section{CONCLUSION}

The three main approaches to chromosome biotechnology are aimed to different but somewhat overlapping target. Using Cre/loxP-system we are able to produce targeted rearrangements of whole chromosomes or their segments or particular genes within the genome, and therefore to modify the set, position and copy number of the endogenous elements of the genome. MACs provide a possibility to introduce into genome relatively large segments of alien chromosome material, either artificially constructed or derived from the genome of different species. Using ES-somatic cell hybrids we may transfer whole chromosomes or their fragments between different genomes within and between species. Each of these approaches has its own advantages and limitations. It seems prospective to combine them to solve particular problems.

\section{ACKNOWLEDGMENTS}

This paper is dedicated to the memory of Prof. Carlos Chagas Filho.

This work was supported by grants from CNPq/PADCT, FAPERJ, Russian Fund of Fundamental Research ((N98-04-49432), Russian Fund "Russian . University for Fundamental Research", and Fund "Gene Engineering and Cell Biology" (N 05.01.04.53).

\section{REFERENCES}

Andrews PW \& Goodfellow PN. 1980. Antigen expression by somatic cell hybrids of a murine embryonal carcinoma cell with thymocytes and L cells. Somat Cell Genet 6: 271-284.

Araki K, Araki M, Miyazaki J \& Vassalli P. 1995. Site-specific recombination of a transgene in fertilized eggs by transient expression of Cre recombinase. Proc Natl Acad Sci USA 92: 160-164.

Babinet C. 1997. Transgenic strategies for the study of mouse development: an overview. In HoudEBINE
LM, editor. Transgenic Animals. Generation and Use. Amsterdam: Overseas Publishers Association, pp 371-386.

Brown W, Heller R, Loupart M-L, Shen MH \& Chand A. 1996. Mammalian artificiL CHROMOSOMES. Curr Opin Gen Dev 6: 281-288.

Brown WR, Mee PJ \& Hong Shen M. 2000. Artificial chromosomes: ideal vectors? Trends Biotechnol 18: 218-223.

Bunting M, Bernstein KE, Greer JM, CAPecchi MR \& Thomas KR. 1999. Targeting genes for selfexcision in the germ line. Genes Develop 13: 1524 1528.

CAPECCHI MR. 1989. Altering the genome by homologous recombination. Science 244: 1288-1292.

CHAN AWS. 1999. Transgenic animals: Current and alternative strategies. Cloning 1: 25-46.

Dieken ES, EpNer EM, Fiering S, Fournier REK \& Groudine M. 1996. Efficient modification of human chromosomal alleles using recombination-proficient checken/human microcell hybrids. Nature Genet 12: 174-182.

Dietrich W, Katz H, Lincoln SE, Shin HS, Friedman J, Dracopoli NC \& LANDER ES. 1992. A genetic map of the mouse suitable for typing intraspecific crosses. Genetics 131: 423-447.

Donovan P. 1994. Growth factor regulation of mouse primordial germ cell development. Curr Top Dev Biol 229: 189-225.

Endo A, Watanabe T \& Fujita T. 1991. An XXY sex chromosome anomaly in the mouse. Genome 34: 41-43.

Farr CJ, Bayne RAL, Kipling D, Mills W, Critcher R \& CoOKe HJ. 1995. Generation of a human Xderived minichromosome using telomere-associated chromosome fragmentation. EMBO J 14: 54445454.

Gmur R, Solter D \& Knowles BB. 1980. Independent regulation of $\mathrm{H}-2 \mathrm{~K}$ and $\mathrm{H}-2 \mathrm{D}$ gene expression in murine teratocarcinoma somatic cell hybrids. $J$ Exp Med 151: pp 1349-1359. 
GoRdON JW. 1997. Transgenic technology and laboratory animal science. ILAR J 38: 32-41.

Gu H, Marth JD, Orban PC, Mossmann H \& RajewSKY K. 1994. Deletion of a DNA polymerase beta gene segment in $\mathrm{T}$ cell using cell type specific gene targeting. Science 265: 103-106.

Hammer RE, Pursel VG, Rexroad CE, Wall RJ, Bolt KM, Ebert RD, Palmiter RD \& Brinster RL. 1985. Production of transgenic rabbits, sheep pigs by microinjection. Nature 315: 680 .

Harrington JJ, Van Bokkelen G, Mays RW, GusTASHAW K \& Willard HF. 1997. Formation of de novo centromeres and construction of firstgeneration human artificial microchromosomes. Nature Genet 15: 345-355.

Heller R, Brown KE, Burgtorf C \& Brown WRA. 1996. Mini-chromosomes derived from the human $\mathrm{Y}$ chromosome by telomere directed chromosome breakage. Proc Acad Sci USA 93: 7125-7130.

Herault Y, Rassoulzadegan M, Cuzin F \& Duboule D. 1998. Engineering chromosomes in mice through targeted meiotic recombination (TAMERE). Nat Genet 20: 381-384.

HERNANDEZ D \& Fisher EMC. 1999. Mouse autosomal trisomy: two's company, three's a crowd. Trends in Genetics 15: 241-247.

Hernandez D, Mee PJ, Martin Je, Tybulewicz VLJ \& Fisher EMC. 1999. Transchromosomal mouse embryonic stem cell lines and chimeric mice that contain freely segregating segments of human chromosome 21. Human Mol Genet 8: 923-933.

Hogan B, Beddington R, Constantini F \& Lacy E. 1994. Manipulating the Mouse Embryo. 2.ed. New York: Cold Spring Harbor Laboratory Press, p. 497.

Lasko M, Pichel JG, Gorman JR, Sauer B, Okamoto Y, Lee E, Alt FW \& Westrhal H. 1996. Efficient in vivo manipulation of mouse genomic sequences at the zygote stage. Proc Natl Acad Sci USA 93: 58605865 .

Lewandoski M \& Martin GR. 1997. Cre-mediated chromosome loss in mice. Nature Genet 17: 223225 .
Matveeva NM, Shilov AG, Bayborodin SI, PhilimoNenko VV, Rolinskaya IV \& Serov OL. 1996. Hybrid between mouse embryonic stem and somatic cells retain pluripotency. Proc Acad Sci Russia 349: 129-132.

Matveeva NM, Shilov AG, Kaftanovskaya HM, BAYBorodin SI, FoKina MM, MaXIMOVSKy LP, Zhelezova AI, Golubitsa AN \& Serov OL. 1998. In vitro and in vivo study of pluripotency in hybrid cells obtained by fusion of mouse embryonic stem cells with splenocytes. Mol Reprod Development 50: 128-138.

Metzger D \& Feil R. 1999. Engineering the mouse genome by site-specific recombination. Current Opinion in Biotechnology 10: 470-476.

Miller RA \& Ruddle FH. 1977. Properties of teratocarcinoma-thymus somatic cell hybrids. Cell 9: $45-55$

PAlmiter RD \& Brinster RL. 1986. Germ-line transformation of mice. Ann Rev Genet 20: 465-499.

Papaionnnou V \& Johnston R. 1993. Production of chimeras and genetically defined offspring from targeted ES cells. In: Joyner AL, editor. Gene Targeting. A Practical Approach. Oxford: Oxford University Press, pp 107-146.

Ramirez-Solis R, Liu P \& Bradley A. 1995. Chromosome engineering in mice. Nature 378: 720-724.

Robertson EJ. 1987. Embryo-derived stem cell lines. In: ROBERTSON RJ, editor. Teratocarcinomas and Embryonic Stem Cells: A Practical Approach. Oxford: IRL Press Limited, pp 71-112.

Schwenk F, BARON U \& Rajewsky K. 1995. A cretransgenic mouse strain for the ubiquitous deletion of loxP-flanked gene segments including deletion in germ line. Nucleic Acid Res 23: 5080-5081.

Serov OL, Zhdanova NS, Pack SD, Lavrentieva MV, Shilov AG, Rivkin MI, Matyakhina LD, Draber P, Kerkis AY, Rogozin IB \& Borodin PM. 1990. The mink $\mathrm{X}$ chromosome: organization and inactivation. In: Ogita Z \& C MARkert (eds). Isozymes: Structure, Function, and Use in Biology and Medicine. Progress in Clinical and Biological Research, v. 344, New York: Willey-Liss, pp 589-619. 
Serov OL, Matveeva NM, Shilov AG, KaFtanovskaya HM, KuZnetsov SB \& Zhelezova AI. 1999. Transfer of the $X$ chromosome derived from somatic cells into the murine genome by embryonic stem x somatic cell hybridization. In: INTERNATIONAL SYMPOSIUM ON X CHROMOSOME INACTIVATION IN MAMMALS. Novosibirsk, Russia. Proceedings of International Symposium on $X$ chromosome inactivation in mammals. Novosibirsk, N. Broockdorf, 1999, p.21-22.

Smith AJH, de Sousa MA, Kwabi-Addo B, HeppellParton A, Impey H \& Rabbits P. 1995. A sitedirected chromosomal translocation induced in embryonic stem cells by Cre-loxP recombination. $\mathrm{Na}$ ture Genet 9: 376-385.

Tada M, Tada T, Lefebvre L, Barton SC \& Surani MA. 1997. Embryonic germ cells induce epigenetic reprogramming of somatic nucleus in hybrid cells. EMBO J 3: 6510-20.

Takagi N, Yoshida Ma, Sugawara O \& Sasaki M. 1983. Reversal of $X$ inactivation in female mouse somatic cells hybridized with murine teratocarcinoma stem cells in vitro. Cell 34: 1053-1062.
TAKAGI N. 1988. Requirement of mitoses for the reversal of X-inactivation in cell hybrids between murine embryonal carcinoma and normal thymocytes. Expl Cell Res 175: 363-375.

Thomas KR \& CAPECCHI MR. 1987. Site-directed mutagenesis by gene targeting in mouse embryo-derived stem cells. Cell 51: 503-512.

Tomizuka K, Yoshida H, Uejima H, Kugoh H, Sato K, Ohguma A, Hayasaka M, Hanaoka K, Oshimura M \& IsHidA I. 1997. Functional expression and germline transmission of a human chromosome fragment in chimaeric mice. Nat Genet 16: 133-143.

Tomizuka K, Shinohara T, Yoshida H, Uejima H, Ohguma A, Tanaka S, Sato K, Oshimura M \& IsHIDA I. 2000. Double trans-chromosomic mice: maintenance of two individual human chromosome fragments containing heavy and kappa loci and expression of fully human antibodies. Proc Natl Acad Sci USA 97: 722-727.

Van Deursen J, Fornerod M, Van Rees B \& Grosveld G. 1995. Cre-mediated site-specific translocation between nonhomologous mouse chromosomes. Proc Natl Acad Sci USA 92: 7376-7380. 